\title{
RHEUMATIC SUBCUTANEOUS NODULE FORMATION
}

\author{
By F. DUDLEY HART
}

A WEALTH of literature has sprung up, particularly in the last few years, dealing with subcutaneous nodule formation in acute rheumatism and rheumatoid arthritis, but there is still much difference of opinion as to whether the two are closely related or whether the tissue changes are non-specific and unrelated ætiologically. As Keil (1938) writes, in a very full and comprehensive analysis of the literature on the subject, the alleged relation between rheumatic fever and rheumatoid arthritis has been adduced principally on the basis of (1) the occasional resemblances in their clinical features, including the occurrence of subcutaneous nodules; (2) the similarities in pathological changes seen in the tissues about the joints and in the subcutaneous lesions; (3) data revealed by immunological methods, which, however, are still in the early stages. There are many facts which suggest that the two conditions are of similar ætiology, but clinically the differences between the two conditions are marked, and workers have pointed out histological differences in the structure of the nodules in the two conditions (Collins, 1937).

This last author quotes the work of Massell, Mote, and Jones (1937), as does also Keil, who suggests that their work be verified using similar methods of control. These workers noted the importance of trauma in nodule formation in rheumatism, and attempted to reproduce these lesions by injections of the patient's own blood into the subcutaneous and deeper tissues of the elbow. For the next ten days the patient was instructed to rub the injected elbow on the bedclothes for some minutes six times a day. By these means they produced nodules two to three weeks later which they found clinically indistinguishable from those seen in rheumatic fever, and were thus able to study the different stages in nodule formation by histological examination after biopsy. Eighty-two patients suffering from rheumatic fever or chorea 
were taken and thirty-four other non-rheumatic subjects used as controls. One elbow was injected as above, and the other was used as a second type of control. In some cases saline was injected, in others novocain with and without friction, in others friction alone, in others blood alone without friction. In the group of eighty-two patients so treated thirty-seven (45 per cent.) grew nodules, while in the thirty-four non-rheumatic controls only one developed a definite nodule, and in this case it was thought that the subject might have a rheumatic carditis. Friction alone produced no nodules in any subject, rheumatic or otherwise. Novocain in no case produced nodules, whether friction was applied or not. Injection of blood alone produced nodules occasionally, but not nearly so often as when friction was applied. Saline, however, caused nodules to grow in four patients out of twenty where whole blood in the opposite elbow had produced positive results in six cases. The authors considered that this was due to bleeding from torn vessels in the injected area, but this should presumably have also occurred to some extent with novocaine. Histologically they claimed that the lesions so produced were very similar to true rheumatic nodules, the differences lying in the infiltration of blood cells and phagocytosis present in their cases. They considered the stages of nodule formation to be very briefly: (1) alteration in the structure of the collagen with resultant œdema and deposition of fibrin-like material; (2) concurrently or immediately afterwards vascular damage with polymorphonuclear and lymphocytic infiltration and proliferation of the primitive perivascular mesenchyme and other cells, these invading the collagen foci; (3) gradual organisation, starting from perivascular areas towards the centres of the areas of necrosis; and, concurrently, (4) a typical cellular reaction-closely packed basophilic mononuclear and multinuclear cells staining like fibroblasts and apparently capable of depositing intercellular collagen. The outcome was a progressive organisation to a normal fibrous tissue replacement of the lesion. The lesions were therefore remarkably similar to rheumatic nodules, though the identity was by no means established.

The results obtained might be supposed to have been due to trauma alone and/or to actual deposition in the tissues of a nodule-forming substance present in the blood of rheumatic subjects. It would seem that-if this work be verifiedhere might be the basis of a study of tissue changes in 
rheumatism from which fresh facts might possibly arise which would throw new light on the rtiology of the disease. We therefore attempted to reproduce this work on a group of rheumatic, rheumatoid and normal subjects. The patients were in the wards of the Royal Northern Hospital and West Wickham Heart Hospital, and may be grouped thus:

(a) Numbers 1 to 10 were over the age of thirty and suffering from rheumatoid arthritis. None had a history of rheumatic fever or chorea, none had a heart lesion. Two had sedimentation rates of under $15 \mathrm{~mm}$. in one hour (micro-method, normal one to eight), eight a reading above this number. Three had subcutaneous nodules over the elbows. Eight were women, two men.

(b) Numbers 11 to 20: Active rheumatism in children under fifteen years of age. All had evidence of rheumatic carditis. Six had typical subcutaneous rheumatic nodules. In all the sedimentation rate was raised.

(c) Numbers 20 to 34: Subacute rheumatism in children under the age of fifteen years. All had passed through the acute phase, but still had raised sedimentation rates. All but one had, or had had, signs of carditis. Eight had had nodules within three months of examination which had disappeared.

(d) Numbers 34 to 50: Rheumatism in children under fifteen years with no signs of activity. Children convalescent and getting up. All had been considered to have a carditis. Sedimentation rates normal.

(e) Numbers 50 to 54: Cases of Still's disease, one inactive, three with signs of activity.

As a control a series of children suffering from other nonrheumatic conditions was used, but in only eight were injections given, as owing to the negative results in the rheumatic series this seemed to become unnecessary.

Technique. - 3 c.c. of blood was withdrawn from the median cubital vein and reinjected over the right olecranon process subcutaneously. This produced a large bulge over the point of the elbow and slightly distally, which became painless within a few minutes. The patients were instructed to massage the area vigorously several times a day with the other hand. No anæsthetic was used.

Over the left elbow 3 c.c. of normal saline was given in like manner in each case. In those cases where nodules were present over the elbows the malleoli were used or, in three cases, the 
tibial processes. The latter is, however, rather an unsuitable area, but had to be used, as both elbows and malleoli were already involved in these cases.

In the first eight cases plasma alone was injected, as it was thought that this would lessen the complexity of the histology of any resulting nodule. When it was seen that there seemed to be no resultant nodule formation, this was stopped and whole blood given. The blood of four active cases actually forming nodules was injected into eight other cases-two rheumatoids, two controls, one Still's disease and three active rheumatics. Blood from one case of Still's disease and three of rheumatoid arthritis was injected into four subacute and two active rheumatic patients.

The result was entirely negative. The patients were watched for the next five weeks and no nodules of our making appeared. In two cases where we injected blood over the internal malleolus Nature provided them within a week over the external, but in no case over the experimental area. As no nodules were formed we did not feel justified in making biopsies of the tissues infiltrated.

\section{Discussion}

It is difficult to explain the different results obtained by the American workers. If trauma were considered the most important factor, sufficient trauma should have been obtained in our cases, as 3 c.c. of blood raised quite a considerable hæmatoma. The total fluid injected was less by 1 c.c. in our cases, as no novocaine was given. The friction was carried out religiously in those cases under direct supervision, but it is likely that in many children the time spent on rubbing was only a few minutes a day. Nevertheless, no different results were obtained in twelve cases who were known to persevere with their exercises and were seen constantly throughout the day. It seemed that in our series of cases nodules could not be produced experimentally, though several were in a nodule-forming phase or had been so in the preceding few weeks. In five cases nodules appeared in areas other than those injected in the subsequent five weeks, so it is likely that on the law of averages we would have come across a nodule eventually in an area previously injected. Collins states that a type of fibrinoid necrosis can occur as an effect of trauma 
and we have seen nodules appear in rheumatic subjects after trauma, but this seems to occur only at certain times in certain subjects and we have been unable to reproduce it experimentally.

\section{SUMMARY}

An attempt was made to reproduce rheumatic nodules by injection into rheumatic subjects over bony points of $(a)$ the patient's whole blood, $(b)$ whole blood from active nodule-forming cases of rheumatism, (c) separated blood plasma, $(d)$ whole blood from rheumatoid subjects, and $(e)$ saline, in each case friction being applied to the affected part. Four groups of patients were used: forty rheumatic (active, subacute and inactive), ten rheumatoid, four Still's disease and eight nonrheumatic subjects. No nodules were reproduced experimentally in any case, although in some cases new nodules formed elsewhere as part of the normal disease process.

My thanks are due to Dr. Bernard Schlesinger for his invaluable help, and to the nursing staff of the Royal Northern and West Wickham Heart Hospitals for their kind co-operation in the above work.

\section{REFERENCES}

Collins, D. H. (1937): Journ. Path. Bact., 45, 97.

KFIL, H. (1938): Medicine, 17, 309.

Masseld, B. F., Mote, J. R., and Jones, T. D. (1937): Journ. Clin. Invest., $16,125$. 